\title{
Performance of ALICE silicon tracker detector
}

\section{G. Luparello* for the ALICE Collaboration}

\section{NIKHEF, Amsterdam.}

E-mail: gluparel@cern.ch

\begin{abstract}
ALICE (A Large Ion Collider Experiment) is the LHC experiment devoted to the study of the strong interacting matter created in heavy-ion collisions. The ALICE Inner Tracking System (ITS) consists of six layers of silicon detectors exploiting three different technologies: pixel, drift and strip (from inside to outside). It covers the central pseudorapidity range, $|\eta|<0.9$, and its distance from the beam line ranges from $r=3.9 \mathrm{~cm}$ for the innermost pixel layer up to $r=43 \mathrm{~cm}$ for the outermost strip layer. The main tasks of the ITS are to reconstruct the primary and secondary vertices, to track and identify charged particles with a low- $p_{\mathrm{T}}$ cutoff and to improve the momentum resolution at high $p_{\mathrm{T}}$. During the operations, the ITS has demonstrated its tracking and vertexing capabilities, which are in excellent agreement with the design values. In these proceedings, after a brief description of the features of the system, the performance during the first three years of data taking at LHC will be presented.
\end{abstract}

22nd International Workshop on Vertex Detectors,

15-20 September 2013

Lake Starnberg, Germany

\footnotetext{
* Speaker.
} 


\section{Introduction}

ALICE is a multi-purpose heavy-ion experiment designed to study the physics of strongly interacting matter and the quark-gluon plasma formed in nucleus-nucleus collisions at LHC. The physics program includes also the study of proton-proton and proton-nucleus collisions as a benchmark measurement for the heavy-ion program. Thanks to the features of the experimental apparatus, like the low momentum cut-off, the excellent particle identification capability and the efficient minimum bias trigger, ALICE developed also a detailed pp collision program to complement the observations of the dedicated pp experiments at LHC.

The ALICE detector is composed of a central barrel, covering the full azimuth in the central pseudorapidity region $(|\eta|<0.9)$, and a forward $(2.5<\eta<4.0)$ muon arm [1]. The Inner Tracking System (ITS) is the innermost detector of the central barrel. Together with the Time Projection Chamber (TPC), the ITS provides a robust tracking in a wide momentum range (100 MeV/c $100 \mathrm{GeV} / \mathrm{c}$ ) in the high density track environment produced in central $\mathrm{Pb}-\mathrm{Pb}$ collisions. A good tracking resolution at high $p_{\mathrm{T}}$ is guaranteed by a large level arm, while the multiple scattering effects are reduced by minimizing the total material budget $\left(13 \% X_{0}\right)$. The detectors of the central barrel provide also particle identification (PID) in a wide $p_{\mathrm{T}}$ range through different techniques: $\mathrm{d} E / \mathrm{d} x$, time-of-flight, transition radiation and Cherenkov radiation.

A detailed description of the ITS hardware will be given in Section 2. In Section 3, the ITS performance during the first three years of data taking at LHC (Run 1) will be discussed. Finally, in Section 4, the ITS capability for vertexing, tracking and particle identification will be described.

\section{System overview}

The Inner Tracking System (ITS) is the innermost detector of ALICE. It is composed of six cylindrical layers exploiting three different technologies: pixel, drift and strip. The position and segmentation of the six layers of the ITS and the technology were chosen to limit the occupancy to a few $\%$ in the high multiplicity environment expected for central $\mathrm{Pb}-\mathrm{Pb}$ collisions and to guarantee an efficient tracking. The main parameters of each layer of the ITS are summarized in Table 1. The geometrical layout of the ITS layers is shown in Fig. 1.

\begin{tabular}{|c|c|c|c|c|c|c|}
\hline Layer/Type & $1 / \mathrm{SPD}$ & 2/SPD & 3/SDD & 4/SDD & $5 / \mathrm{SSD}$ & 6/SSD \\
\hline$r[\mathrm{~cm}]$ & 3.9 & 7.6 & 15.0 & 23.9 & 38.0 & 43.0 \\
\hline $\pm z[\mathrm{~cm}]$ & 14.1 & 14.1 & 22.2 & 29.7 & 43.1 & 48.9 \\
\hline N. of modules & 80 & 160 & 84 & 176 & 748 & 950 \\
\hline Channels & 3276800 & 6553600 & 43008 & 90112 & 1148928 & 1459200 \\
\hline $\begin{array}{c}\text { Active area per module } \\
r \phi \times z\left[\mathrm{~mm}^{2}\right]\end{array}$ & \multicolumn{2}{|c|}{$12.8 \times 70.7$} & \multicolumn{2}{|c|}{$70.2 \times 75.3$} & \multicolumn{2}{|c|}{$73 \times 40$} \\
\hline Intrinsic resolution $r \phi, z[\mu \mathrm{m}]$ & \multicolumn{2}{|c|}{12,100} & \multicolumn{2}{|c|}{35,25} & \multicolumn{2}{c|}{20,830} \\
\hline Material budget $X / X_{0}$ & 1.14 & 1.14 & 1.13 & 1.26 & 0.83 & 0.83 \\
\hline
\end{tabular}

Table 1: Inner Tracking System parameters. 


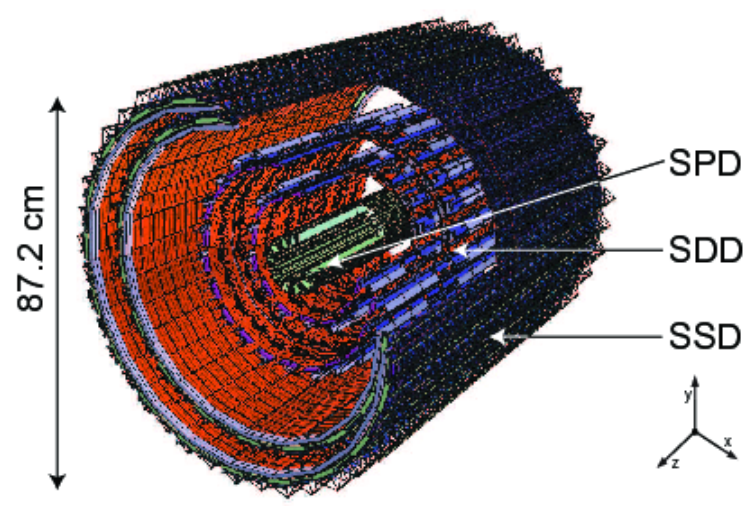

Figure 1: Layout of the Inner Tracking System.

The two internal layers are equipped with Silicon Pixel Detectors (SPD) and are placed at mean radii of 3.9 and $7.6 \mathrm{~cm}$, respectively. The radius of the most internal SPD layer is the minimum allowed by the beam-pipe. The innermost SPD layer has an extended pseudorapidity coverage $(|\eta|<1.98)$ to provide a larger acceptance for the measurement of charged-particles multiplicity. The basic building block of the SPD is a module consisting of a two-dimentional sensor matrix of reversed biased silicon detector diodes, $200 \mu \mathrm{m}$ thick, bump-bonded to 5 front-end chips, thinned down to $150 \mu \mathrm{m}$. The pixel size is $50 \mu \mathrm{m}(r \phi) \times 425 \mu \mathrm{m}(z)$. It is normally operated at $\sim 50 \mathrm{~V}$ with a measured leakage current less than $2 \mu \mathrm{A}$. Two modules are mounted together along the $z$ direction to form an half-stave. In total the SPD is composed by 60 staves, consisting of 240 modules. The SPD has a binary readout and provides a prompt (Fast-OR) trigger signal contributing to the Level0 trigger of ALICE [2].

The two intermediate layers are equipped with Silicon Drift Detectors (SDD) and are placed at mean radii of 15.0 and $23.9 \mathrm{~cm}$. This was a compromise to have precise bi-dimensional points with a reduced number of channels. Each SDD module consists of a drift sensor and its front-end electronics. The sensitive area of a sensor is split into two drift regions, with electrons moving in opposite $(r \phi)$ directions, by a central cathode kept at a nominal voltage of $-1800 \mathrm{~V}$. In each drift region, and on both detector surfaces, $291 \mathrm{p}+$ cathode strips, with $120 \mu \mathrm{m}$ pitch, fully deplete the detector volume and generate a drift field of $\sim 500 \mathrm{~V} / \mathrm{cm}$ parallel to the wafer surface. A second medium voltage supply of $-40 \mathrm{~V}$ keeps the biasing of the collecting region independent of the drift voltage, and directs the electron charges toward the collecting anodes. Each drift region is equipped with 256 anodes with $294 \mu \mathrm{m}$ pitch (along the $z$ direction) to collect the charges, and three rows of 33 MOS charge injectors used in monitoring the drift velocity as a function of the anode. The two-dimensional points are reconstructed exploiting the anode segmentation in the $z$ direction and the drift time in $r \phi$. The modules are mounted on linear structures called ladders. There are 14 ladders with six modules each on the inner SDD layer and 22 ladders with eight modules each on the outer SDD layer for a total of 260 modules.

The last two layers are equipped with Silicon Strip Detectors (SSD) at mean radii of 38.0 and $43.0 \mathrm{~cm}$. The last SSD plane was placed as close as possible to the TPC in order to achieve a high track matching efficiency between ITS and TPC. Each SSD module is composed of a 1536-strip 
double-sided sensor connected to twelve front-end chips. The strips have $95 \mu \mathrm{m}$ pitch and are oriented on the $\mathrm{P}(\mathrm{N})$-side of the sensor at an angle of 7.5(27.5) mrad with respect to the beam $(z)$ direction. The orientation of the strips, approximately parallel to the magnetic field provided by the ALICE solenoid magnet, ensures that the best position resolution is achieved in the bending direction. The small stereo angle of the strips on the modules ensures a low probability for double coincidences, which generate "ghost" hits. The modules are assembled on ladders, as for the SDD. The innermost SSD layer is composed of 34 ladders, each of them being an array of 22 modules. The outermost SSD layer is composed by 28 ladders each made of 25 modules for a total of 1698 modules.

The SDD and SSD have analogue readout and therefore can be used for particle identification via specific energy loss $(\mathrm{d} E / \mathrm{d} x)$ in the non relativistic region.

The ITS contributes to the ALICE detector performance with the following tasks: the primary vertex reconstruction, the improvement of the impact parameter resolution with a precision better than $100 \mu \mathrm{m}$ to detect the secondary vertices produced by the decays heavy-flavour mesons, the improvement of momentum resolution for particles reaching the TPC and also the standalone tracking and PID capability for low momentum particles.

\section{ITS operations during LHC Run 1}

The ALICE ITS performance were almost stable during the first three years of data taking independently from the colliding system ( $\mathrm{pp}, \mathrm{p}-\mathrm{Pb}$ and $\mathrm{Pb}-\mathrm{Pb}$ ) and the beam energy. At the end of the operations before the shut down in February 2013, about 90\% of the ITS modules were used for the data acquisition. For both the SDD and the SSD the number of modules in acquisition was almost constant over time.

For the SPD the percentage of operable staves varied with time, and in particular increased after having reestablished the coolant flow. During the whole commissioning phase preceding the installation of the detector inside the ALICE experimental hall, the system showed full efficiency $(100 \%)$ and a mean temperature of the sensors of $28^{\circ} \mathrm{C}$ with an input pressure before the capillaries of 2.7 bar and no problem or misbehavior was observed. Each sector was individually tested and a thermal survey with an infrared camera was also performed [3]. After the installation in ALICE, the detector showed the first problems with $50 \%$ of one of the SPD sectors switched off due to high temperature. After that, also other sectors started to show the same behavior, with the cooling flow clearly decreasing in the lines. Some attempts of increasing the flow by increasing the liquid pressure were only partially successful and did not allow the full recovery of the performance. The flow in some lines continuously kept decreasing despite the pressure rising, until only $60 \%$ of the detector could be switched on at the end of 2011. Test campaigns [4] demonstrated that the misbehavior was connected to the partial clogging of the filters located along the cooling lines, $1.5 \mathrm{~m}$ away from the detector, in order to protect the capillaries from pollution. The filter clogging caused a decrease of the flow and thus an increase of the pressure. Attempts to clean the filters by counterflow flushing and ultra-sound showed very low effectiveness. Since the filters were not reachable without a partial dismounting of ALICE, an ad-hoc drilling procedure was developed [4]. This intervention allowed restoring the nominal flow rate in the lines of the SPD cooling system signif- 
icantly improving the efficiency of the detector. After the drilling intervention at the beginning of the 2012, 111 of the 120 half staves were operational.

The three ITS subdetectors are operated as independent systems in the experimental area. Therefore, each subdetector implemented its own tools to operate the hardware and to online monitor the quality of the collected data. In addition, each ITS subdetector developed an independent calibration procedure.

The remote control of the hardware is done through the Detector Control System (DCS) that allows the operation of the different detectors by means of an interactive and customizable interface. The ITS control system is integrated in the ALICE DCS [5] and is organized into four sub-projects: the SPD, SDD, SSD control systems for the three sub-detectors, and the PIT control system for the pixel trigger system. In addition to the remote operation of the hardware, the DCS allows also the monitoring and archiving of the working parameters, the implementation of interlocks and alarms to protect the equipments agains potentially dangerous conditions.

The online monitoring of the quality of the data is performed through the Data Qualiy Monitoring (DQM). The DQM software system, named AMORE (Automated MOnitoRing Environment), has been developed specifically for the ALICE experiment [6]. AMORE is based on a publishersubscriber paradigm where a large number of processes, called agents, execute detector-specific decoding and analysis of raw data samples and publish their results in a pool. Clients can then connect to the pool and display the monitoring histograms through a dedicated user interface. The monitoring of these published histograms allows for a prompt individuation of detector deconfigurations during data taking that would influence the quality of the data. The monitored objects vary for each detector. For the SPD the parameters controlled are the hitmaps per chip in each SPD layer, the Fast-Or bit map, the correlation between the number of fired Fast-Or in the inner layer and outer layer per event. In addition, summary plots to identify data format errors or multi event buffer errors are present. For the SDD the monitored objects are the module occupancy of the two layers. For the SSD the distribution of the data size in the event and the module occupancy are monitored.

\section{Calibration procedures}

The calibration procedures developed for each ITS subdetector are described in the following. These procedures are based on standalone runs taken usually at the beginning of the LHC fill and aim at the individuation of the dead and noisy channels and the measurement of the gain factor for the SDD.

- SPD: the main part of the SPD calibration was done before the installation and consisted in the search of optimal thresholds and parameters of the FEE to obtain an uniform response of the pixel matrices. This configuration turned out to be highly stable and therefore the only calibration procedure requested regularly is the search of noisy channel. The presence of noisy channels can have influences on the trigger capability, therefore several procedures have been developed to identify noisy channels. Two types of standalone runs are launched without beam: the first one is triggered by a fixed frequency clock and identify channels constantly noisy, the second one uses the self triggering mode to identify channels that are noisy at a different rate. The noisy pixels found in this way are masked at the level of the FEE. 
An additional search for noisy pixels is done during the data-taking. In this case, a dedicated Detector Algorithm processes the SPD data and mark a pixel as noisy if it fires more than 5\% of the events. The amount of noisy pixels spotted in this way is very low: if present, they are of the order of a unity. The map of noisy pixels created by the Detector Algorithm is stored in the Offline Condition Database (OCDB) and retrieved during the offline reconstruction. An online search for noisy pixels is also implemented in the DQM.

- SDD: the calibration procedure for the SDD is based on three types of standalone runs: pedestal, pulser and injector runs. The pedestal runs measure the baseline signal and identify the noisy channels. For all anodes the run-by-run fluctuation of the baselines are below 1.5\%. The average noise is $\sim 2.3$ ADC counts, matching the design value and small compared to the 100 ADC counts of the charge peak for a MIP particle crossing the sensor close to the anodes. The baselines are stored in the FEE and used to equalize the signals. The pulser runs measure the gain for each electronic channel equalizing the signals of the anodes when calculating the collected charge of the SDD reconstructed points. The distribution of the gain for all the anodes in acquisition has an r.m.s. of $\sim 4 \%$ demonstrating a good uniformity of the response of the electronic channels. In general the gain turned out to be quite stable during the data-taking, with run-by-run fluctuations within $2 \%$. The injector runs provide a measurement of the drift speed as a function of the anode coordinates by exploiting the MOS injectors implanted on the detector surface. The accurate knowledge of the drift speed at the level of $\sim 0.1 \%$ is important to reach the requested $35 \mu \mathrm{m}$ spatial resolution. The two main contributions to the drift speed variations are the temperature $\left(v_{\text {drift }} \propto T^{-2.4}\right)$ and the non uniformity of the drift field (i.e. non linearity in the voltage divider or inhomogeneity in dopant concentration). While the latter has been mapped in laboratory with laser measurements [7], the former needs continuous measurements and temperature stability within $0.1 \mathrm{~K}$. These calibration runs are collected periodically during the data-taking and analyzed by dedicated Detector Algorithms that stores the obtained calibration parameters in the OCDB from where are then retrieved during the offline reconstruction.

- SDD: the SSD calibration is based on pedestal runs taken with a $5 \mathrm{~Hz}$ trigger rate in absence of beam at the beginning of each LHC fill. The pedestal run includes the calculation of the baselines and the intrinsic noise of each channel. The typical noise value is of 2.4 and 3.5 ADCs depending of the sensor side considered. On the basis of the pedestal measurements, the quality of each channel is assessed and the parameters computed at the end of the run by a dedicated Detector Algorithm are stored in the OCDB. The noise values are also used to set the Zero Suppression thresholds, which allows transferring the physics signals with an on-the-fly suppression of noise data. The gain factor is calculated at a sensor-side level and is based on the offline analysis of the detector response to minimum ionizing particles crossing the silicon. Since this calibration turned out to be very stable, it was performed for the first time at the very beginning of the data taking and in the subsequent periods only small updates were needed. The gain factor calculated offline is stored in the OCDB and used at the reconstruction level. The run-by-run fluctuations are $\sim 2 \%$. The gain calibration is also a useful tool to spot hardware defects: strong deviations from the equalized response, 
generally caused by suddenly misbehaving hybrids or single chips, can be spotted run-by-run and fixed by masking the faulty hardware in the data acquisition.

\section{Physics performance}

In this section, the performance of the ITS from the point of view of the vertexing, tracking and particle identification are described. In order to achieve the demanding requirements in terms of tracking efficiency and impact parameter resolution a very accurate alignment and description of the material budget are fundamental. The ITS is made of 2198 sensors that have to be aligned. The alignment was performed before the beginning of the data-taking and monitored during the operations. The alignment procedure used the optical and mechanical survey measurements as a starting point. Then, a first alignment was performed with cosmic ray tracks collected in 2008 [8] and refined with a sample of pp tracks at $7 \mathrm{TeV}$ collected in 2010 with and without magnetic field.

In ALICE, the ITS plays a crucial role in the computation of the interaction primary vertex. Two algorihms are based on the informations provided by the ITS: the VertexerSPD $z$ and the VertexerSPD3D. The first one provides a measurement of the $z$ coordinate of the interaction point, using the average location of the collisions region in the transverse plane. The second one provides a 3-dimentional measurement of the interaction point, using the SPD tracklets in a fiducial window $(\Delta \eta, \Delta \phi)$. The ITS vertexing algorithms provide a quasi-online monitoring of the vertex position and a first estimantion of the vertex for the tracking procedure. Finally, at the end of the tracking procedure a more precise estimation of the interaction vertex is obtained using a stright line approximation of the fully reconstructed tracks in the vicinity of the vertex.

The ITS strongly contributes to the tracking and the points reconstructed in the ITS layers are used to track the charged particles in the central barrel in combination with the other ALICE detectors. The track reconstruction starts in the TPC outermost radius where the track density is minimal and continues inward matching TPC reconstructed tracks to the SSD external layer toward the inner ITS layers and the primary vertex. Afterwards the tracks are backpropagated through the ITS, TPC and the outer detectors (TOF, HMPID, PHOS, EMCal) for particle identification. A further refit is performed inwards to refine the tracks parameters. Finally the primary vertex is recalculated with optimal resolution by using the reconstructed tracks. The ITS points leftover after the global tracking procedure are used for the ITS-standalone-tracking, which aims at extending the $p_{\mathrm{T}}$ acceptance down to $\sim 100 \mathrm{MeV} / c$ and reconstructing the high-momentum tracks going in dead zones of the TPC. The tracking precision of the system can be assessed through the impact parameter resolution, that is a convolution of the track-position and primary vertex resolution. The impact parameter $\left(d_{0}\right)$ is defined as the distance of closest approach of a given track from the primary vertex. In Fig. 2 the resolution of the transverse component of the impact parameter is shown for tracks reconstructed in the central barrel and satisfying the standard track quality tracks. The resulting impact parameter resolution is better than $65 \mu \mathrm{m}$ at $p_{\mathrm{T}}>1 \mathrm{GeV} / c$ almost independently from the colliding system and is in agreement with the expected values from Monte Carlo simulations within 10\%. The impact parameter resolution is slightly better for $\mathrm{Pb}-\mathrm{Pb}$ collisions due to better resolution achieved in the primary vertex reconstruction.

The ITS identifies low-momentum particles in the non-relativistic region measuring the energy loss $\mathrm{d} E / \mathrm{d} x$ in SDD and SSD silicon. The four analogue measuremens of the charge collected in 


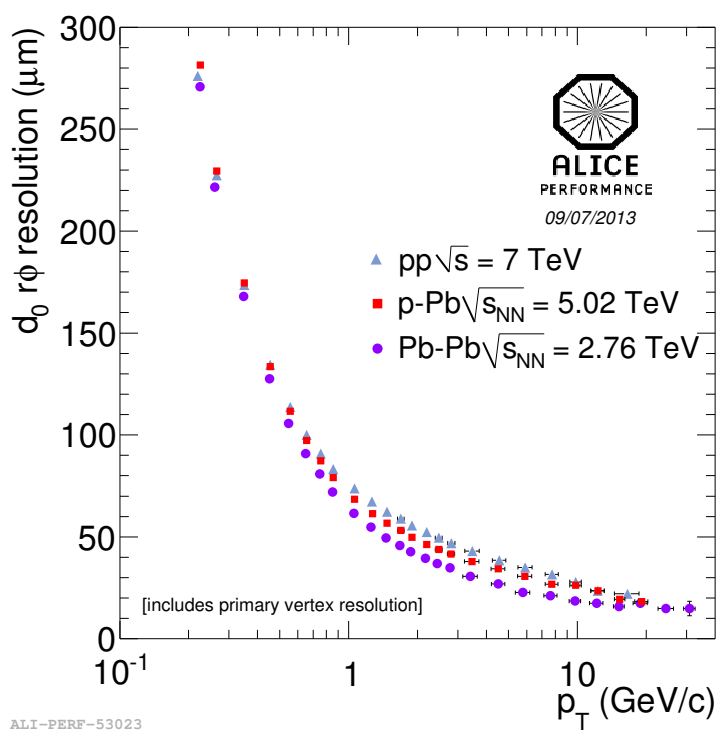

Figure 2: Impact parameter resolution as a function of $p_{\mathrm{T}}$ for tracks reconstructed in the central barrel for three collision systems: pp collisions at $7 \mathrm{TeV}$ (triangles), $\mathrm{Pb}-\mathrm{Pb}$ collisions at $2.76 \mathrm{TeV}$ (squares) and $\mathrm{p}-\mathrm{Pb}$ collisions at $5.02 \mathrm{TeV}$ (circles).

the four layers are combined by means of the trunkated mean method. The bands of kaons, pions and protons detected in $\mathrm{Pb}-\mathrm{Pb}$ collisions are visible in Fig. 3 (left panel) centered around the continuous lines representing the theoretical curves from the Bethe-Bloch parametrization of the energy loss. The ITS achieve an energy resolution of 10-15\% (see Fig. 3, right panel) and allows separating kaons from protons up to $1 \mathrm{GeV} / \mathrm{c}$ and pions up to $450 \mathrm{MeV} / c$, covering also the region below $100 \mathrm{MeV} / c$, outside of the TPC and TOF particle identification capability.
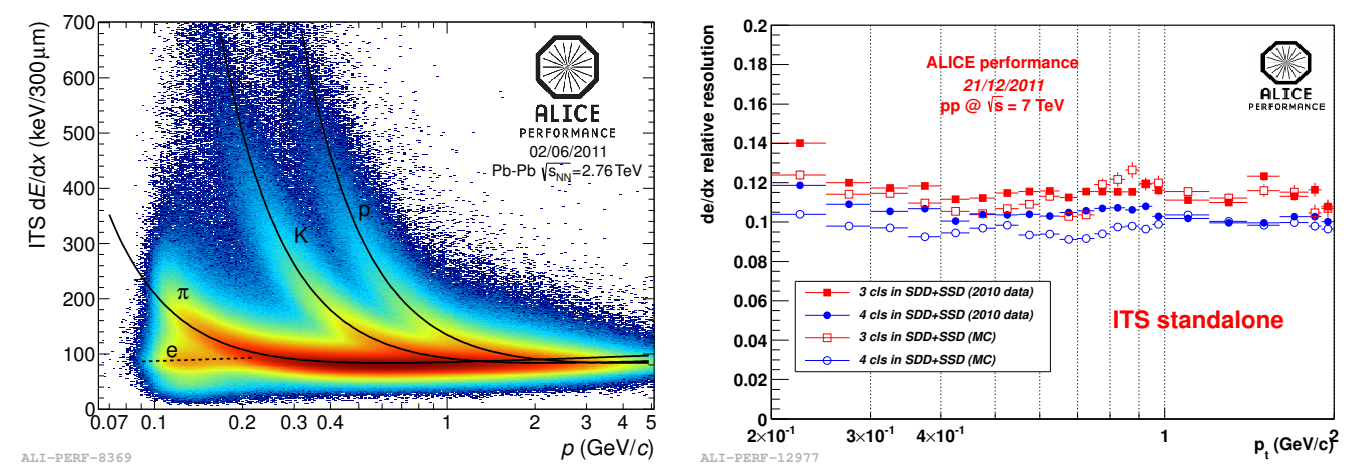

Figure 3: Left: The $\mathrm{d} E / \mathrm{d} x$ of charged particle as a function of their momentum measured in $\mathrm{Pb}-\mathrm{Pb}$ collisions at $2.76 \mathrm{TeV}$. Right: Relative resolution for $\mathrm{d} E / \mathrm{d} x$ of charged particles as a function of the momentum in pp collisions at $7 \mathrm{TeV}$ for 3 (red squares) and 4 (blue triangles) clusters in the ITS compared with Monte Carlo expectations (open markers). 


\section{Summary}

The Inner Tracking System of the ALICE experiment was operated successully and with remarkable stable performance during the first three years of data taking in $\mathrm{pp}, \mathrm{p}-\mathrm{Pb}$ and $\mathrm{Pb}-\mathrm{Pb}$ collisions. The ITS contributed significantly to the event reconstruction, matching more than $90 \%$ of the tracks reconstructed by the TPC and improving momentum and angular resolutions. In standalone mode it extended tracking and PID capabilities of the experiment down to $p_{\mathrm{T}}>1 \mathrm{MeV} / c$ by reconstructing the tracks missed by the TPC. The ITS provided also a prompt reconstruction of the interaction primary vertex. Therefore the ITS played a crucial role in the ALICE data analysis. In order to improve the ITS physics performance and in particular to extend heavy quark studies to the decays of charmed baryons and beauty hadrons, an upgrade of the system is under development [9].

\section{References}

[1] ALICE Collaboration, The ALICE experiment at the CERN LHC, 2008 JINST 3 S08002

[2] G. Aglieri Rinella et al., The pixel trigger system for the ALICE experiment, PoS (RD09) 005 (2009)

[3] A. Pepato et al., The mechanics and cooling system of the ALICE silicon pixel detector, Nucl. Instrum. Methods Phys. Research A 565 (2005) 6âĂŞ12

[4] A. Francescon et al., Performance of the ALICE SPD cooling system, J. Phys.: Conf. Ser. 395 (2012) 012063

[5] P. Chochula et al., IEEE Transactions on Nuclear Science (2010), vol. 57, 472-478

[6] B. von Haller et al., The ALICE Data Quality Monitoring System, J. Phys.: Conf. Ser. 331 022030, 2011

[7] F. Prino et al., Characterization of the ALICE Silicon Drift Detectors using an infrared laser, JINST, 3 P06004 (2008)

[8] ALICE Collaboration, Alignment of the ALICE Inner Tracking System with cosmic-ray tracks, JINST, 5 P03003 (2010)

[9] ALICE Collaboration, Upgrade of the Inner Tracking System Conceptual Design Report, CERN-LHCC-2012-013; LHCC-P-005 